\title{
Dependence of Non-Linear Impairments on Polarization State and Baud Rate in WDM Systems with Mixed DQPSK and OOK Channels
}

\author{
Mohsan Niaz Chughtai ${ }^{1}$, Marco Forzati ${ }^{1}$, Jonas Mårtensson ${ }^{1}$, Danish Rafique ${ }^{2}$ \\ 1) Acreo Netlab, Electrum236, SE16440Kista, Sweden \\ Tel.:+46(0) 86 327804,E-mail:mohsan.niaz@acreo.se \\ 2) Photonic Systems Group, Tyndall National Institute and Department of EE /Physics, \\ UCC, Cork, Ireland, E-mail:danish.rafique@tyndall.ie
}

\begin{abstract}
In this paper, transmission of Polarization-Multiplexed (PM), RZ-DQPSK is analyzed numerically with copropagation of intensity modulated non return to zero on off keying (NRZ OOK) channels. The dependence of nonlinear crosstalk on baud rate $(10 \mathrm{Gbaud}, 28 \mathrm{Gbaud}$ and $56 \mathrm{Gbaud})$ and relative state of polarization (SOPs) of PM RZ DQPSK and neighbouring NRZ OOK channels is analyzed in terms of required OSNR for the BER of $10^{-3}$ versus launch power. From the results we observe that non-linear impairments are highly dependent on relative SOPs and less dependent on baud rates of the PM RZ DQPSK test channel.
\end{abstract}

\section{INTRODUCTION}

In recent years a lot of research has been carried out for the investigation of efficient multilevel modulation schemes for increasing the spectral efficiency of fiber optical communication systems in terms of $\mathrm{b} / \mathrm{s} / \mathrm{Hz}$. This includes both direct and coherent detection at the receiver [1]. However, the performance of these phase modulated optical communication systems is seriously degraded by nonlinear effects, especially self-phase modulation (SPM) and cross-phase modulation (XPM) [2]. In particular it has been shown that XPM arising from the neighbouring pre-deployed intensity-modulated lower-bit-rate channels is a major source of performance degradation [3], [4].

In a previous paper [5][6] we have numerically investigated the performance of PM RZ-DQPSK transmission at $40 \mathrm{Gbaud}$, in the presence of neighbouring channels modulated by $10 \mathrm{~Gb} / \mathrm{s} \mathrm{NRZ} \mathrm{OOK}$. In this paper we numerically investigate the impact of baud rate as well as the relative SOP of PM RZ DQPSK and NRZ OOK channels. The transmitter set-up, transmission link and receiver, are shown in Figure 1 for simulations using VPItransmissionMaker ${ }^{\circledR}$. The impact of nonlinear cross talk from NRZ OOK neighbouring WDM channels on the PM RZ DQPSK modulated test channel is analyzed in terms of required OSNR for bit error rate (BER) of $10^{-3}$ as a function of launch power.

\section{SIMULATION SETUP}

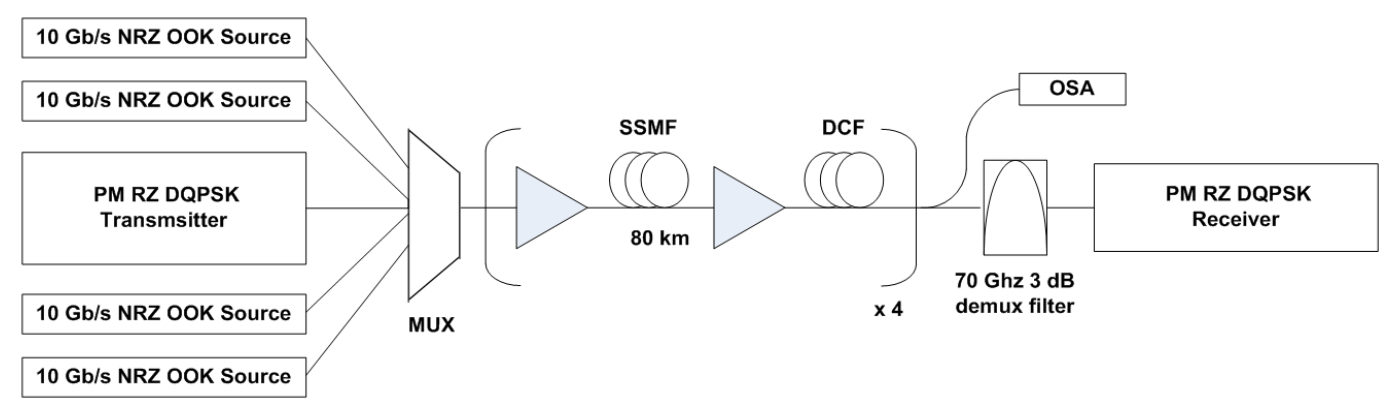

Figure 1. Experimental setup for simulations.

The PM RZ DQPSK transmitter (see Fig. 2) was driven by De-Bruijn bit sequence (DBBS) of order 13 for X-polarization and DBBS sequence of order 12 for y-polarization to maintain data de correlation in the two polarizations. For the carrier a laser source (1 MHz linewidth) at wavelength of $1550 \mathrm{~nm}$ was used. The output signal is combined by a polarization beam combiner $(\mathrm{PBC})$ and then pulse carved at $50 \%$ duty cycle by an extra MZM.

The optical fiber link consists of four spans of $80 \mathrm{~km}$ standard single mode fiber (SSMF) amplified by erbium doped fiber amplifiers (EDFAs). The dispersion was compensated up to $95 \%$ in each span by dispersion compensating fibers (DCFs). The attenuation in SSMF was $0.2 \mathrm{~dB} / \mathrm{km}$ and the dispersion was $17 \mathrm{ps} / \mathrm{nm}-\mathrm{km}$. For the WDM system the neighbouring NRZ OOK channels were driven by different (PRBS) sequences at $10 \mathrm{~Gb} / \mathrm{s}$. The optical amplifiers are modelled as ideal noise-free EDFAs and all optical noise is added at the receiver. Cross-polarization nonlinear effects were included according to the Manakov model [7] $]^{1}$ and PMD effects were neglected. 


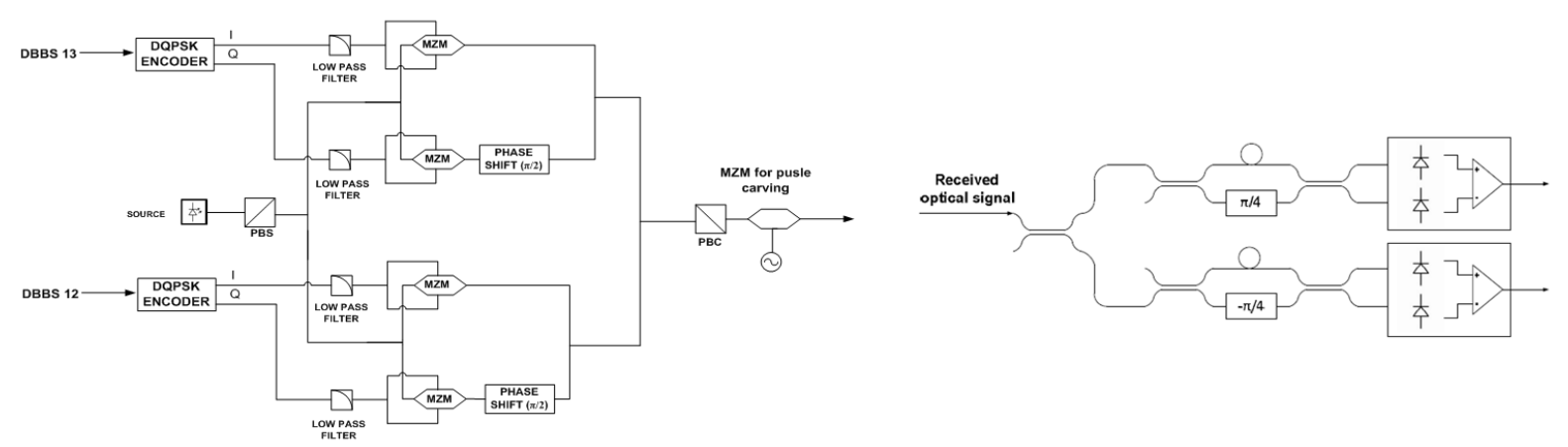

Figure 2. Schematic diagram of DQPSK transmitter and receiver.

At the receiver white Gaussian optical noise is added to the signal (in order to vary the OSNR), before being demultiplexed using a Gaussian filter having a bandwidth of $70 \mathrm{GHz}$. The differential demodulation was done by using delay line interferometers (DLI). The demodulated signal was detected by balanced detectors having $70 \%$ bandwidth of baud rate. The architecture of DQPSK demodulator [8] is shown in Fig. 2. OSNR measurement was done at $0.1 \mathrm{~nm}$. Figure 3 shows the spectrum of the transmitted signal with different PM RZ DQPSK baud rates as central channel having $100 \mathrm{GHz}$ guard band from $10 \mathrm{~Gb} / \mathrm{s}$ neighbouring NRZ OOK channels with $50 \mathrm{GHz}$ separation from each other.

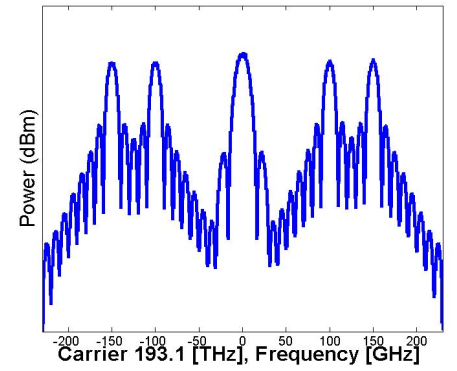

(a)

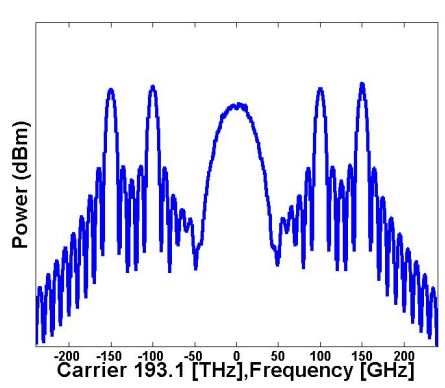

(b)

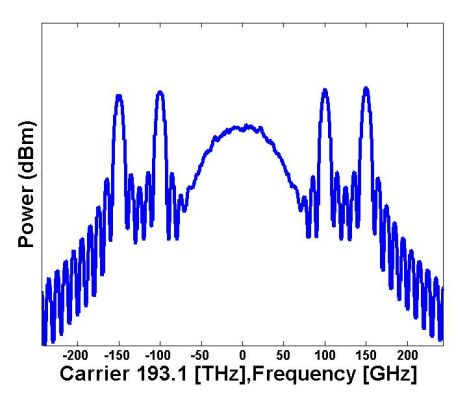

(c)

Figure 3. Spectrum of transmitted signal: 10 Gbaud PM DQPSK (a), 28 Gbaud PM DQPSK (b), 56 Gbaud PM DQPSK (c) with $10 \mathrm{~Gb} / \mathrm{s}$ NRZ OOK channels.

\section{SIMULATION RESULTS and DISCUSSION}

The back-to-back performance is studied in Figure 4, which shows the required OSNR to obtain BER $=10^{-3}$ is around $12.67 \mathrm{~dB}, 15.4 \mathrm{~dB}$ and $18.8 \mathrm{~dB}$ for 10 Gbaud, 28 Gbaud and 56 Gbaud PM RZ-DQPSK, respectively. The difference in required OSNR when moving from 10 Gbaud to 28 Gbaud is $2.73 \mathrm{~dB}$ and that of moving from 28 Gbaud to 56 Gbaud is $3.3 \mathrm{~dB}$.

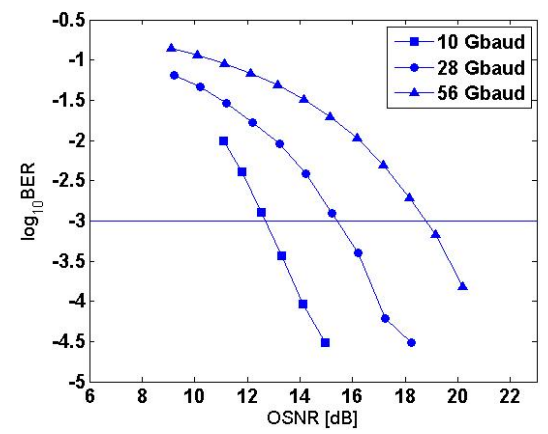

Figure 4. BER as a function of OSNR for back to back transmission of 10, 28 and 56 Gbaud PM RZ DQPSK signal.

Next the results for transmission simulations are shown in Fig. 5 which plots the required OSNR for BER $=10^{-3}$ versus launch power per channel per polarization for two scenarios, one when neighbouring NRZ OOK channels have linear horizontal polarization (LHP) SOP and second when NRZ OOK channels have $+45^{\circ}$ SOP relative to PM RZ DQPSK channel. The total launch power during the simulations was adjusted so that each channel in every polarization and wavelength has equal average launch power.

The impact of nonlinearities is apparent in all the cases since as the launch power increases the required OSNR also increases. For each scenario launch power is increased until BER $=10^{-3}$ is no longer achievable even in the absence of noise. However the required OSNR for relative SOP of $+45^{\circ}$ is higher than that of LHP for all the three baud rates of PM RZ DQPSK channel. In order to understand the source of this penalty the SOPs of the 
received PM RZ DQPSK signal at different launched powers is plotted on Poincaré Sphere for the two cases of $+45^{\circ}$ and LHP relative SOPs in Figure 6 for only 28 Gbaud as they were observed to be same for other baud rates.

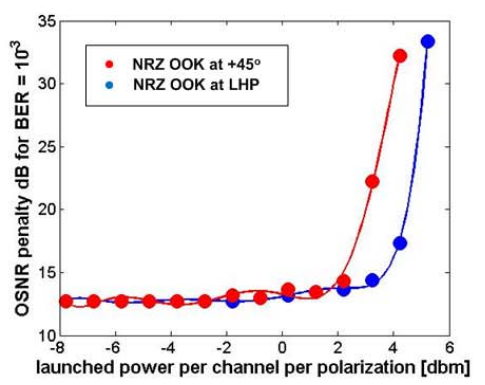

(a)

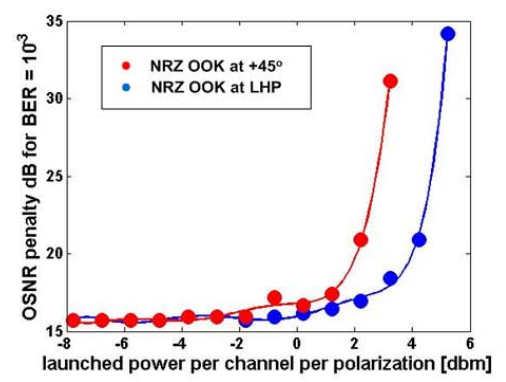

(b)

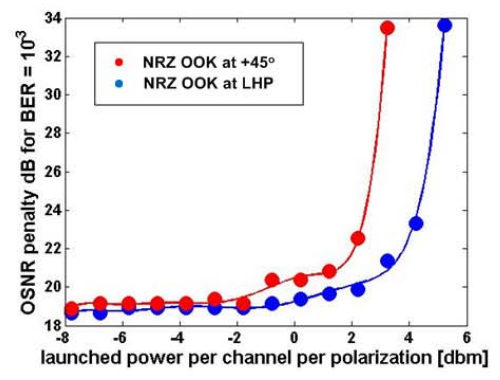

(c)

Figure 5. Required OSNR versus launch power per channel per polarization for PM RZ-DQPSK at

10 Gbaud (a), 28 Gbaud (b) and 56 Gbaud (c) with NRZ OOK channels at LHP and + 45 o relative SOP.

Ideally the PM RZ DQPSK channel has SOPs at $+45^{\circ}$, right hand circular (RHC), $-45^{\circ}$, and Left hand circular (LHC) depending on the relative phase of $x$ and $y$ polarization, but due to non-linear cross talk from neighbouring NRZ OOK channels at LHP and $+45^{\circ}$, the SOPs shift from their ideal positions, indicated by red dots. The blue dots indicate the total Stokes vector as a vector sum of the NRZ OOK and PM RZ DQPSK Stokes vectors.

The plots in Fig. 5 show the results for three cases. For the first case (a) the NRZ OOK channels have LHP, i.e., they are aligned with the $x$ polarization of PM RZ DQPSK signal, this results in rotation of SOP of PM RZ DQPSK along the meridian of $+45^{\circ}, \mathrm{RHC},-45^{\circ}$, and $\mathrm{LHC}$ or around the total stokes vectors at LHP according to the Manakov model [9]. The resulting required OSNR due this rotation is indicated by blue curves in Fig. 6. Similarly in Fig. 6 case (b) when the NRZ OOK channels have $+45^{\circ}$ SOP, the PM RZ DQPSK SOPs rotate along the RHC, LHP, LHC and linear vertical polarization (LVP), or around the total Stokes vector at $+45^{\circ}$. This rotation is compensated by using a wave-plate and resulting SOPs are shown in Fig. 6 case (c). The resulting required OSNR after this rotation is indicated by red curves in Fig. 6.

The reason for the higher required OSNR in case (c) is due to the fact that even after compensation the variance of SOPs is highest along the meridian of RHC, LVP, LHC and LHP which, introduces ellipticity in the SOP of the signal, which when split by a polarization beam splitter (PBS) splitter at the receiver, results in imbalanced amplitudes in $x$ and $y$ polarizations and thus introducing additional errors in the system.

Figure 5 also shows that the dependence of the overall non-linear impairments (intra-channel and crosscannel) on baud rate is low: indeed the non-linear tolerance (NLT, defined as the launch power for which the required OSNR is increased by $1 \mathrm{~dB}$ compared to back-to-back) varies only by $0.5 \mathrm{~dB}$ for LHP and only by $2.2 \mathrm{~dB}$ for $45^{\circ}$, suggesting at higher baud rates that the increase in penalty arising form self phase modulation (SPM) with increasing baud rate (for which dispersion causes pulses to broaden - and hence the power profile to deviate from constant - more quickly) is compensating the decrease in penalties arising from cross phase modulation (XPM) predicted in previous studies [10], [11]. The exact interplay of SPM and XPM is currently being studied. Another noteworthy fact arising in figure 6 is the difference in the scattering behaviour of the signal SOP in the cases of LHP and $+45^{\circ}$ (more symmetric in the former than in the latter case). The explanation may lie in the non-linear SOP rotation taking place in the (dispersion broadened) symbols for which the neighbouring OOK channels are in a 0 state: investigation is currently ongoing.

\section{CONCLUSIONS}

We studied the impact of nonlinearities on PM RZ DQPSK transmission at 10 Gbaud, 28 Gbaud and 56 Gbaud with co propagation of NRZ OOK channels. We observed that the nonlinear cross-talk arising from neighbouring channels is highly dependent on the relative SOP. The nonlinear cross-talk is highest when the relative SOP between the PM RZ DPSK and NRZ OOK channels is $45^{\circ}$. We did not observe a significant impact of the baud rate of the DQPSK channel on total non-linear impairments. 


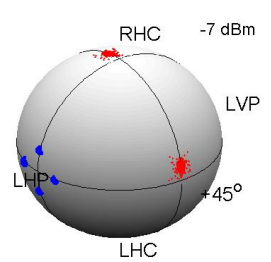

(a)

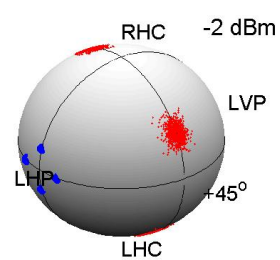

(a)

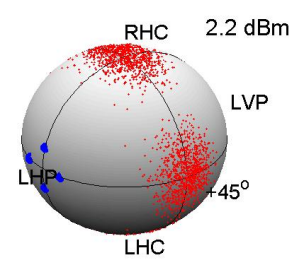

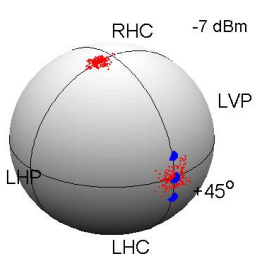

(b)

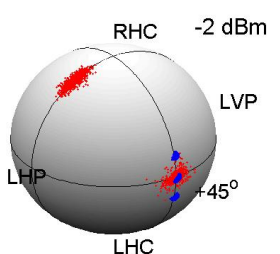

(b)

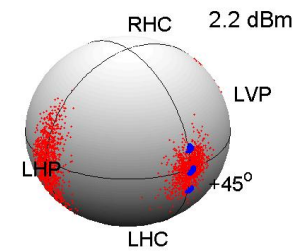

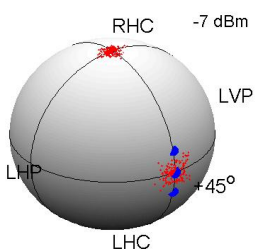

(c)

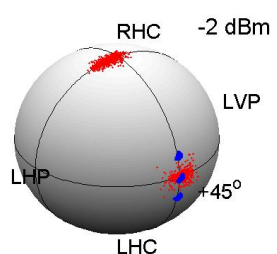

(c)

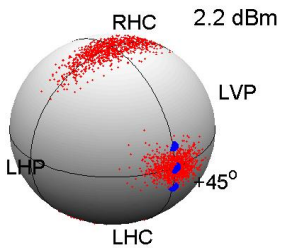

Figure 6. SOP of PM RZ - DQPSK signal as (red dots), after transmission at different launch powers, when the SOP of the NRZ OOK neighboring channels is LHP (a), $+45^{\circ}(b)$; in (c) the signal shown in (b) passed through a wave plate; the blue dots are the vector sum of the Stokes vectors of all the wavelength channels at the transmitter.

\section{ACKNOWLEDGEMENT}

The work described in this paper was carried out with the support of EUROFOS project, Network of Excellence funded by the European Commission through the $7^{\text {th }}$ ICT-Framework Programme.

\section{REFERENCES}

[1] Kahn, J.M.; Keang-Po Ho; "Spectral efficiency limits and modulation/detection techniques for DWDM systems," Selected Topics in Quantum Electronics, vol. 10, no. 2, pp. 259- 272, March-April 2004.

[2] Keang-Po Ho; "Performance degradation of phase-modulated systems due to nonlinear phase noise," Photonics Technology Letters, vol. 15, no. 9, pp. 1213-1215, Sept. 2003.

[3] Jensen, J.B.; Schiellerup, G.; Peucheret, C.; Tokle, T.; Jeppesen, P.; "XPM-induced degradation of multilevel phase modulated channel caused by neighboring NRZ modulated channels," in Proc. OFC/NFOEC 2008, pp .1-3, 24-28 Feb. 2008.

[4] Siano, R.; Marazzi, L.; Boffi, P.; Martelli, P.; Parolari, P.; Righetti, A.; Martinelli, M.; "Cross phase modulation penalties in polarization-multiplexed RZ-DQPSK transmission," in Proc. CLEO/QELS 2006, pp. 1-2, 21-26 May 2006.

[5] Chughtai, M.N.; Forzati, M.; Mårtensson, J.; Tipsuwannakul, E.; Karlsson, M.; , "Analysis of non-linear impairments in 40 Gbaud PM DQPSK and D8PSK transmission," in Proc. ICTON 2010, pp.1-4, June 27- July 12010.

[6] E. Tipsuwannakul, M. Chughtai, M. Forzati, J. Mårtensson, P. Andrekson, and M. Karlsson, "Influence of self- and cross-phase modulation on 40 Gbaud dual polarization DQPSK/D8PSK signals in 10 Gbit/s OOK WDM systems," Opt. Express 18, 24178-24188 (2010).

[7] C. R. Menyuk and B. S. Marks, "Interaction of polarization mode dispersion and nonlinearity in optical fiber transmission systems," J. Lightwave Technol., vol. 24, no. 7, pp. 2806-2826, 2006.

[8] Griffin, R.A.; Carter, A.C.; , "Optical differential quadrature phase-shift key (oDQPSK) for high capacity optical transmission," in Proc. OFC 2002, pp. 367- 368, 17-22 Mar. 2002.

[9] M. Karlsson, and H. Sunnerud, "Effects of nonlinearities on PMD-induced system impairments," J. Lightwave Technol. 24, 4127-4137 (2006).

[10] A. Bononi, M. Bertolini, P. Serena, G. Bellotti, "Cross-phase modulation induced by OOK channels on higher-rate DQPSK and coherent QPSK channels," J. Lightwave Technol. 27, 3974-3983 (2009).

[11] Vassilieva, O.; Hoshida, T.; Rasmussen, J.C.; Naito, T.; , "Symbol rate dependency of XPM-induced phase noise penalty on QPSK-based modulation formats," in Proc. ECOC 2008, pp. 1-2, 21-25, Sep. 2008. 